\title{
Mechanism of myocardial ischemia with an anomalous left coronary artery from the right sinus of Valsalva
}

\author{
Carlo R. Bartoli, PhD, ${ }^{\text {a,b,c }}$ William B. Wead, PhD, ${ }^{\text {a }}$ Guruprasad A. Giridharan, $\mathrm{PhD},{ }^{\text {b,d }}$ \\ Sumanth D. Prabhu, MD, ${ }^{\mathrm{c}}$ Steven C. Koenig, PhD, ${ }^{\text {,d }}$ and Robert D. Dowling, $\mathrm{MD}^{\mathrm{e}}$
}

\begin{abstract}
Objective: An ectopic coronary artery that courses between the aortic root and the pulmonary trunk may lead to sudden cardiac death, especially in athletes. It has been speculated that during exercise, compression of the coronary artery between the great vessels may impair coronary blood flow and produce myocardial ischemia and fatal arrhythmia. However, this hypothesis cannot be tested in humans, and little experimental data exist to explain this phenomenon. To this end, in a calf with an anomalous left coronary artery that coursed from the right sinus of Valsalva between the great vessels, we assessed for myocardial ischemia during pharmacologically induced tachycardia and hypertension.
\end{abstract}

Methods: We identified a juvenile male calf $(103 \mathrm{~kg})$ with an anomalous left coronary artery from the right sinus of Valsalva that coursed between the great vessels. Via thoracotomy, the animal was instrumented for hemodynamic measurements. Intravenous dobutamine increased heart rate and myocardial metabolic demands. Intravenous phenylephrine produced arterial hypertension and increased myocardial metabolic demands. Fluorescent-labeled microspheres were used to map regional myocardial blood flow, and hemodynamics were recorded during each condition. Masson's trichrome staining for fibrosis, wheat-germ agglutinin staining for myocyte size, terminal deoxynucleotidyl transferase dUTP nick end-label staining for apoptosis, and isolectin-B4 staining for capillary density were performed.

Results: For the first time, empiric data documented that an ectopic coronary artery produced myocardial ischemia during elevated myocardial metabolic demands. Left coronary artery resistance increased in a cardiac cycle-dependent pattern that was consistent with systolic compression between the great vessels. Increased cardiac fibrosis, myocyte hypertrophy, cardiac apoptosis, and capillary density indicated that regional ischemic, inflammatory-mediated myocardial remodeling was present.

Conclusions: These findings confirm the proposed mechanism of sudden death and support early surgical repair of coronary arteries that course between the aortic root and the pulmonary trunk. (J Thorac Cardiovasc Surg 2012;144:402-8)

An ectopic coronary artery is a well-described congenital malformation. In 1990, an angiographic survey of 126,595 patients reported that 1 out of 660 humans $(0.15 \%)$ is born with a coronary artery that originates from the opposite sinus of Valsalva. ${ }^{1}$ Most patients are asymptomatic, yet the initial presentation is often sudden cardiac death. ${ }^{1-10}$ Recently, widespread computed

\footnotetext{
From the MD/PhD Program, ${ }^{\text {a }}$ Department of Physiology and Biophysics, University of Louisville School of Medicine, Louisville, Ky; Cardiovascular Innovation Institute, ${ }^{\mathrm{b}}$ University of Louisville, Louisville, Ky; Department of Medicine, ${ }^{\mathrm{c}}$ Institute of Molecular Cardiology, University of Louisville, Louisville, Ky; Department of Bioengineering, ${ }^{\mathrm{d}}$ University of Louisville, Louisville, Ky; and Dowling Consulting, Inc, ${ }^{\mathrm{e}}$ Louisville, Ky.

Funding: American Heart Association Scientist Development Grant 0730319N.

Disclosures: Authors have nothing to disclose with regard to commercial support.

S.C.K. and R.D.D. contributed with equal mentorship of the first author.

Received for publication April 20, 2011; revisions received July 18, 2011; accepted for publication Aug 4, 2011; available ahead of print May 7, 2012.

Address for reprints: Carlo R. Bartoli, PhD, University of Louisville Cardiovascular

Innovation Institute, 302 E. Muhammad Ali Blvd, 4th Floor Louisville, KY 40202

(E-mail: crbart02@louisville.edu).

$0022-5223 / \$ 36.00$

Copyright (c) 2012 by The American Association for Thoracic Surgery doi:10.1016/j.jtcvs.2011.08.056
}

tomograpic angiography has increased the identification of this anomaly. However, guidelines for management have not been well defined, ${ }^{8,9,11}$ in part because of a lack of mechanistic data.

Anatomies in which a coronary artery courses between the aortic root and the pulmonary trunk are associated with the highest mortality. In these patients, investigators have speculated that during exercise, compression of the coronary artery between the great vessels may impair coronary blood flow and produce myocardial ischemia and fatal arrhythmia. ${ }^{2,4}$ However, this hypothesis has not been tested in humans, and little experimental data exist to explain this phenomenon.

To elucidate potential pathophysiologic mechanism(s) of sudden death, we tested the hypothesis that increased coronary resistance from compression of a coronary artery between the great vessels resulted in myocardial ischemia during elevated myocardial metabolic demands. We identified a calf with an anomalous left coronary artery (LCA) from the right sinus of Valsalva that coursed between the aortic root and the pulmonary trunk and investigated whether increased myocardial metabolic demands 


$$
\begin{aligned}
& \text { Abbreviations and Acronyms } \\
& \begin{aligned}
\text { FITC } & =\text { fluorescein isothiocyanate } \\
\text { LCA } & =\text { left coronary artery } \\
\text { LV } & =\text { left ventricular } \\
\text { RV } & =\text { right ventricular } \\
\text { RCA } & =\text { right coronary artery } \\
\text { TUNEL } & =\text { terminal deoxynucleotidyl transferase } \\
& \text { dUTP nick end labeling }
\end{aligned}
\end{aligned}
$$

(increased heart rate or hypertension) resulted in regional myocardial ischemia. Histology was performed to evaluate for regional, ischemic myocardial remodeling.

\section{MATERIALS AND METHODS \\ Surgical Preparation}

The experimental subject received humane care. Experimental procedures followed animal study protocols approved by the University of Louisville Institutional Animal Care and Usage Committee.

A male, Holstein-Jersey mix calf ( $103 \mathrm{~kg}$ ) was studied. The animal was purchased from Oak Hill Genetics (Ewing, Ill). After arrival at the University of Louisville, the animal passed a standard health screening and a 2-week quarantine. Physical examination, transthoracic echocardiography, and laboratory values were unremarkable.

The animal was initially enrolled in a coronary microembolization study. However, in the catheterization laboratory, multiple attempts to selectively engage the LCA trunk were unsuccessful. An aortogram was performed, and it was found that both the right coronary artery (RCA) and LCA emerged from the right sinus of Valsalva. The LCA trunk passed between the pulmonary trunk and the aortic root. The RCA appeared anatomically normal but small and indicated a left-dominant coronary system. Because of the rare anatomy in this animal, we did not proceed with the experimental protocol, and the animal recovered without incident.

Two weeks later, after appropriate approval was obtained, an acute procedure was performed to study the hemodynamics, regional myocardial blood-flow distribution, and gross and microscopic anatomy in this animal. The animal was anesthetized with isoflurane $(3 \%-5 \%)$. Three surface electrocardiographic leads were sutured to the animal's skin. A left thoracotomy was performed in the fourth intercostal space. The animal was anticoagulated with intravenous heparin (100 units $/ \mathrm{kg}$ ). A high-fidelity micromanometer catheter (Millar Instruments, Houston, Tex) was placed to measure aortic blood pressures. A Transit-time ultrasonic flow probe (Transonic Systems Inc, Ithaca, NY) was placed around the distal LCA trunk to measure volumetric coronary blood flow. Silicone catheters (7F) were advanced into the left atrial appendage and descending aorta for serial delivery of $15-\mu \mathrm{m}$ fluorescent-labeled microspheres and reference blood sampling to map regional myocardial blood flow. ${ }^{12,13}$

\section{Experimental Protocol}

Intravenous dobutamine (5-15 $\mu \mathrm{g} / \mathrm{kg} / \mathrm{min})$ was administered to simulate the increasing levels of myocardial metabolic demands during increasing intensity of exercise in order to detect inducible demand ischemia. ${ }^{14}$ Later, phenylephrine $(20-50 \mu \mathrm{g} / \mathrm{min})$ was administered to produce systemic arterial hypertension. ${ }^{15}$ During each simulated physiologic condition, fluorescent-labeled microspheres were injected to map regional myocardial blood flow, ${ }^{12,13}$ and hemodynamic data were collected.

\section{Regional Myocardial Blood Flow Analysis}

During each experimental condition, a separate color of fluorescentlabeled, 15- $\mu \mathrm{m}$ microspheres (IMT Stason Laboratories, Irvine, Calif) were injected into the left atrium and a reference blood sample was simultaneously withdrawn from aorta.

The microsphere technique enabled the precise measurement of regional blood flow in myocardium as follows. In the left atrium, the microspheres mixed with the blood and were ejected into the aorta to disseminate throughout the body to every organ according to the physiologic distribution of blood flow. As the microspheres approached capillaries, they lodged within the smallest pre-capillary arterioles based on regional tissue blood flow patterns.

During microsphere injection, a reference blood-flow sample was drawn from the aorta at a rate of $15 \mathrm{~mL} / \mathrm{min}$ for 100 seconds with a calibrated syringe pump (Harvard Apparatus, Holliston, Mass). The withdrawal sample acted as a reference to determine regional myocardial blood flows in milliliters per minute per gram of tissue. ${ }^{12}$

After euthanasia, the heart was removed and photographed. The ventricles were sectioned into a multilevel map (Figure 1, $A$ and $C$ ). Tissue sections and reference blood samples were sent to IMT Stason Laboratories for automated digestion and counting of fluorescent microspheres with flow cytometry. Regional blood flows were calculated in milliliters per minute per gram of myocardium. The number of counted microspheres in the reference blood sample (known) was compared with the number of microspheres that lodged and were counted in the myocardial sample of interest (known). The ratio between the 2 sphere counts was equal to the ratio between the calibrated rate of aortic withdrawal (known - 15 $\mathrm{mL} / \mathrm{min}$ ) and flow in the tissue of interest (unknown). Regional myocardial blood-flow maps were constructed on a piece-by-piece basis as a percentage change from baseline.

\section{Hemodynamic Data Reduction and Analysis}

Hemodynamic transducers were pre- and post-calibrated against known physical standards to ensure measurement accuracy. Data were collected at $400 \mathrm{~Hz}$, signal conditioned, and A/D converted for digital analysis with our Good Laboratory Practice-compliant data acquisition system. ${ }^{16}$

To determine hemodynamic values during each physiologic condition, pressure and coronary flow waveforms were used to derive aortic systolic and diastolic blood pressures, and mean and peak systolic and diastolic coronary blood flows. Hemodynamic values were calculated on a beat-to-beat basis for each 30-second data set with the Hemodynamic Evaluation and Assessment Research Tool program ${ }^{17}$ developed in MATLAB, version 6.5 (MathWorks, Natick, Mass). All analyzed beats in each data set ( $\sim 40-90$ beats/30-second data set) were averaged to obtain a single representative mean value. We defined systolic coronary resistance as the ratio of mean systolic blood pressure to mean systolic coronary flow and diastolic coronary resistance as the ratio of mean diastolic blood pressure to mean diastolic coronary flow. During the transition from normal baseline blood pressure to hypertension, volumetric coronary blood flow and coronary resistance were reported as a percentage change from baseline (Figure 1, $D$ and $E$ ).

\section{Histology}

Histology was performed on samples from left ventricular (LV) and right ventricular (RV) myocardium (Figure 2, $C-J$ ) and compared with established bovine values. ${ }^{18}$ Paraffin-embedded tissue sections $(4 \mu \mathrm{m})$ were deparaffinized, rehydrated, and stained. Regional myocardial fibrosis was quantified with Masson's trichrome staining as the ratio of area occupied by collagen stain to the area of myocardium sampled. Regional myocyte size was determined with fluorescein isothiocyanate (FITC)-conjugated wheat-germ agglutinin and DAPI (Molecular Probes, Grand Island, NY) nuclear co-staining to delineate the cell membrane in 100 cross-sectional 


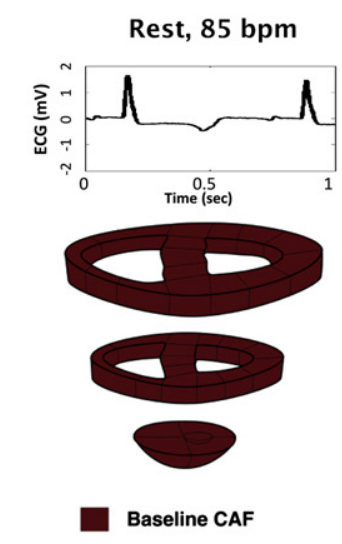

A

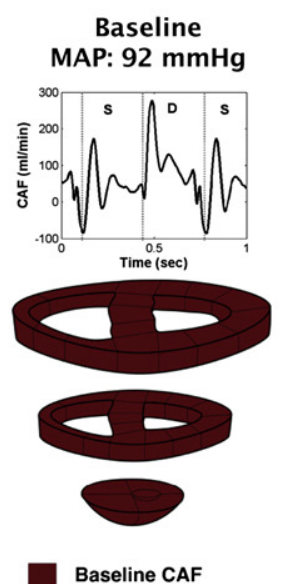

C
Elevated HR, 175 bpm

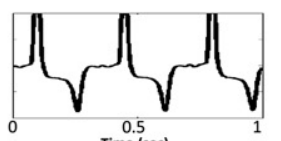
Time (sec)

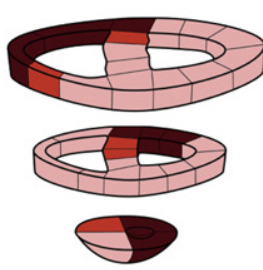

$>\mathbf{3 0 0} \%$ increased flow $\mathbf{2 5 0}-\mathbf{3 0 0} \%$ increased flow $<250 \%$ increased flow

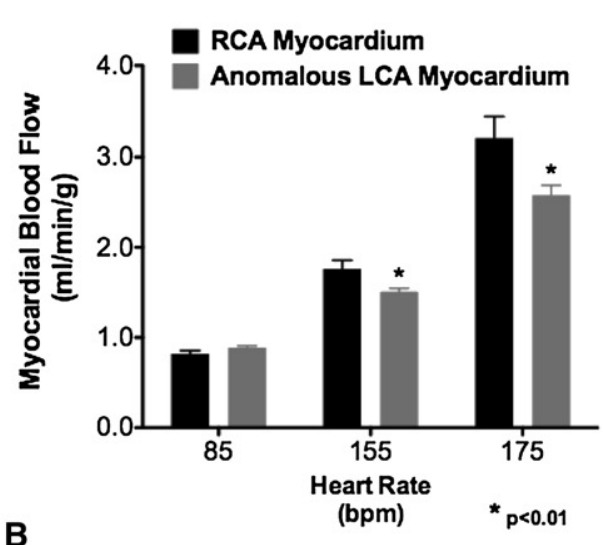

Hypertension MAP: $124 \mathrm{mmHg}$

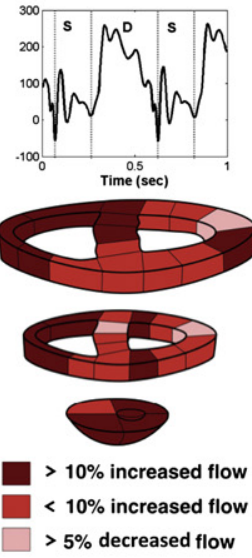

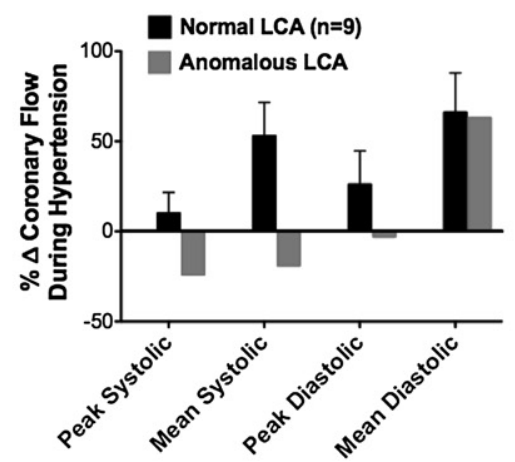

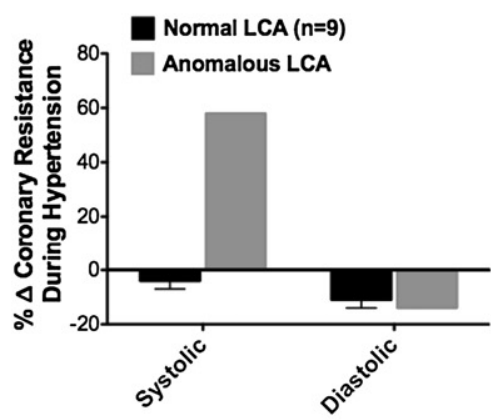

D

E

FIGURE 1. Regional myocardial blood flow was measured in a bovine heart with an LCA that emerged from the right sinus of Valsalva and coursed between the great vessels. A, Lead II electrocardiographic tracings demonstrated ST-segment depression that worsened with increasing heart rate. Regional myocardial blood-flow mapping of the ventricles demonstrated a large area of myocardial ischemia. B, At rest, LV myocardial blood flow was greater than RV myocardial blood flow $(P=.06)$. At elevated heart rates this trend reversed, and blood flow to myocardium supplied by the anomalous LCA was significantly lower than to myocardium supplied by the normal RCA $(* P<.01)$. C, During the transition from normal blood pressure to hypertension, volumetric LCA blood flow tracings showed abnormal decreases in peak and mean systolic and peak diastolic coronary blood flows versus during baseline blood pressure. Similarly, myocardial blood-flow maps showed that, during the hypertension condition, blood flow to LCA myocardium did not increase appropriately and decreased in some regions. Myocardium supplied by the normal RCA showed a normal increase in blood flow during hypertension. $\mathrm{D}$, In control calves $(\mathrm{n}=9)$, hypertension increased coronary blood flow during all phases of the cardiac cycle. However, in the present animal, hypertension decreased systolic and beginning diastolic coronary blood flows. E, The abnormal coronary blood-flow profiles shown in $\mathrm{C}$ and $\mathrm{D}$ were caused by a large, inappropriate increase in coronary resistance during systole. This abnormal pattern of cardiac cycle-dependent coronary resistance and blood flow demonstrated a pattern consistent with systolic aortic root compression of the LCA. $H R$, Heart rate; $L C A$, left coronary artery; $R C A$, right coronary artery; $C A F$, coronary artery flow; $M A P$, mean arterial pressure; $S$, systole; $D$, diastole; $b p m$, beats per minute. $* P<.01 \mathrm{LCA}$ vs RCA myocardial sections.

cells with centrally located nuclei. Regional apoptosis in cardiac tissue was determined with the DeadEnd Fluorometric TUNEL System (Promega Corp, Madison, Wis), which catalytically incorporates fluorescein-12dUTP at DNA strand breaks in cells actively undergoing programmed cell death. Nuclei were counterstained with DAPI. Regional myocardial capillary density was determined with FITC-conjugated isolectin-B4 staining of endothelium as the ratio of area occupied by isolectin-B4 stain to the area of myocardium sampled. Images were viewed with epifluorescence microscopy (Nikon TE2000; Nikon Corp, Tokyo, Japan) and analyzed with Metamorph Imaging Software (Molecular Devices Inc, Sunnyvale, Calif).

\section{Control Animals}

For comparison, control calves with normal coronary anatomy $(n=9)^{15}$ were studied. Animals were instrumented via thoracotomy for hemodynamic coronary blood flow measurement during pharmacologically induced hypertension with phenylephrine (20-50 $\mu \mathrm{g} / \mathrm{min})$.

\section{Statistics}

GraphPad Prism version 4.0 (GraphPad Software Inc, La Jolla, Calif) was used to perform statistical analyses and plot data. Microsphere tissue pieces were separated into territory supplied by the LCA and RCA. An 

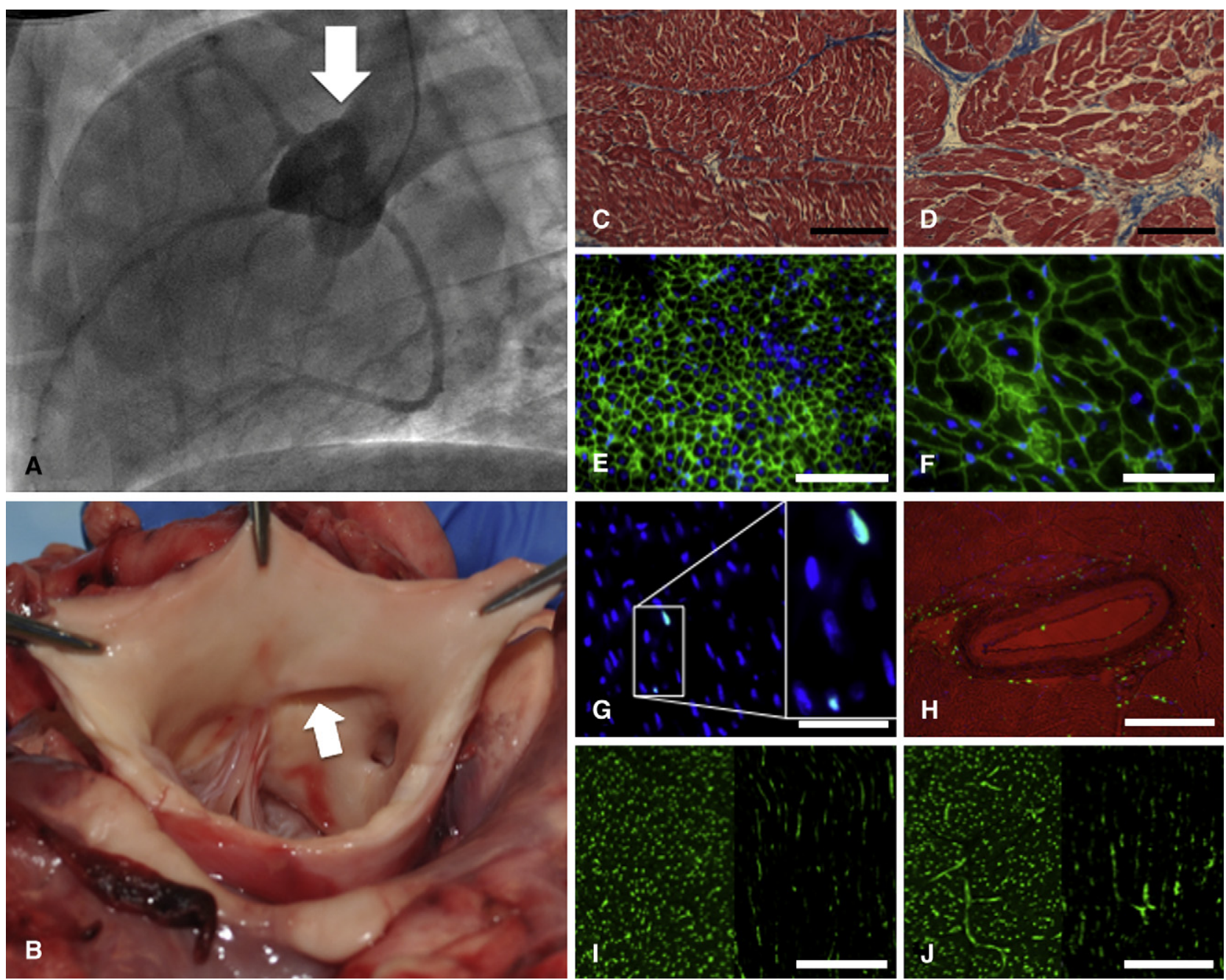

FIGURE 2. A, In the catheterization laboratory, an aortogram revealed an ectopic LCA that emerged from the right sinus of Valsalva. B, Gross examination from within the aortic root demonstrated that the ostium of the LCA originated at an acute angle from the right aortic sinus (arrow). A normal RCA ostium is visible in the right sinus of Valsalva. C and D, Masson's trichrome staining exhibited normal levels of fibrosis in RV myocardium (C) and elevated fibrosis and myocyte hypertrophy in LV myocardium. 40×, black bar $=100 \mu \mathrm{m}$. E and F, FITC-conjugated wheat-germ agglutinin staining confirmed normal sized myocytes in RV myocardium (E) and grossly hypertrophic myocytes in LV myocardium (F). 40×, white bar $=100 \mu \mathrm{m}$. G and H, TUNEL staining revealed an elevated apoptotic rate throughout the heart. G, Two TUNEL ${ }^{+}$nuclei are seen in close proximity in a small sample of LV myocardium. DAPI nuclear stain and FITC TUNEL ${ }^{+}$nuclear stain overlay. $40 \times$, white bar $=100 \mu \mathrm{m} . \mathrm{H}$, Numerous $\mathrm{TUNEL}^{+}$cells are noted in close proximity to a large-caliber intramural coronary artery and vein: Bright field and DAPI nuclear stain and FITC TUNEL ${ }^{+}$nuclear stain overlay. $2 \times$, white bar $=1000 \mu \mathrm{m}$. I and J, Endothelial staining of transverse and longitudinal myocardial sections with FITC-conjugated isolectin-B4 demonstrated higher capillary density and more collateral channels (longitudinal vessels in the transverse plane) in LV myocardium (J) than in RV myocardium (I), which suggested ischemia-induced neovascularization. $10 \times$, white bar $=400 \mu \mathrm{m}$.

unpaired, 2-tailed Student $t$ test was performed to compare differences in myocardial blood flow between the 2 territories (Figure 1,B). All data are presented as mean \pm standard error.

\section{RESULTS}

\section{Myocardial Blood Flow}

At a baseline heart rate, blood flow to myocardium supplied by the anomalous LCA was greater than that supplied by the RCA (Figure 1, B: 85 beats/min; LCA $0.89 \pm 0.02$ vs RCA $0.82 \pm 0.04 \mathrm{~mL} / \mathrm{min} / \mathrm{g}, P=.06$ ). The electrocardiogram was normal, and myocardial blood flow was likely unimpaired at baseline (Figure 1, A). However, as heart rate increased, blood flow to the LCA myocardium was $20 \%$ lower than in the RCA myocardium (Figure 1, B: 155 beats $/ \mathrm{min}, \mathrm{LCA} 1.51 \pm 0.05$ vs RCA $1.76 \pm 0.08$ $\mathrm{mL} / \mathrm{min} / \mathrm{g}, P<.01 ; 175$ beats/min, LCA $2.58 \pm 0.08 \mathrm{vs}$ RCA $3.21 \pm 0.24 \mathrm{~mL} / \mathrm{min} / \mathrm{g}, P<.01)$. Regional bloodflow mapping depicted impaired myocardial blood flow that worsened as heart rate increased (Figure 1, A). Electrocardiograms recorded ST-segment depression that worsened at higher heart rates in a dose-dependent fashion (Figure 1, A). 
To investigate the mechanism, we tested the hypothesis that increased coronary resistance impaired LCA flow. In control calves with normal coronary anatomy $(\mathrm{n}=9),{ }^{15}$ the transition to arterial hypertension (mean arterial pressure: untreated baseline $90 \pm 3 \mathrm{~mm} \mathrm{Hg}$, treated $124 \pm 2$ $\mathrm{mm} \mathrm{Hg}$ ) increased coronary blood flow during all phases of the cardiac cycle. However, in the present animal with the coronary anomaly, hypertension (mean arterial pressure: untreated baseline $92 \mathrm{~mm} \mathrm{Hg}$, treated $124 \mathrm{~mm} \mathrm{Hg}$ ) increased coronary resistance and decreased LCA blood flow during systole and beginning diastole in a pattern that was consistent with aortic root compression (Figure 1, $C-E$ ).

\section{Gross Findings}

Gross examination from within the aortic root demonstrated that the left sinus of Valsalva did not contain a coronary ostium. The ostium of the LCA originated with a slit-like opening at an acute angle from the right aortic sinus (Figure 2, $B$, arrow). A short aortic intramural segment was present just above the anterior aortic valve commissure, and the anomalous LCA assumed an oval shape until the bifurcation of the left anterior descending and left circumflex coronary arteries. Approximately 1.5 $\mathrm{cm}$ of the anomalous trunk coursed between the aortic root and the pulmonary trunk. A normal right ostium was present in the right sinus of Valsalva.

\section{Histopathology}

Regional myocardial remodeling was present in the distribution of the anomalous LCA. Masson's trichrome staining revealed elevated fibrosis in LV myocardium (Figure 2, $C$ and $D$ : LV 6.8\%, RV 4.9\%, vs normal control LV 3.5\% $\pm 1.1 \%$, RV $4.6 \% \pm 0.8 \%{ }^{18}$ ). LV myocytes appeared hypertrophic. Wheat-germ agglutinin staining also demonstrated myocyte hypertrophy in LV myocardium (Figure 2, $E$ and $F$ : myocyte size: $\mathrm{LV} 467 \pm 19 \mu \mathrm{m}^{2}, \mathrm{RV}$ $99 \pm 3 \mu \mathrm{m}^{2}$ vs normal control LV $186 \pm 40 \mu \mathrm{m}^{2}$, RV 112 $\left.\pm 6 \mu \mathrm{m}^{2}\right) .{ }^{18}$ Terminal deoxynucleotidyl transferase dUTP nick end labeling (TUNEL) staining demonstrated abnormal numbers of apoptotic nuclei throughout the heart (Figure 2, $G$ and $H$ : LV $1.2 \%$, RV $0.3 \%$ vs normal control LV $0.04 \% \pm 0.02 \%$, RV $0.15 \% \pm 0.09 \%) .{ }^{18}$ Isolectin-B4 staining of endothelium demonstrated increased capillary density and collateralization in LV myocardium (Figure 2, $I$ and $J$ : LV $21 \%$, vs RV $13 \%$ ).

\section{DISCUSSION}

For the first time, these data documented that an ectopic coronary artery produced myocardial ischemia during elevated myocardial metabolic demands (Figure 1). LCA resistance increased in a cardiac cycle-dependent pattern that was consistent with systolic aortic root compression.

\section{Anomalous Coronary Blood Flow Physiology}

In normal mammals at rest, LV myocardial blood flow exceeds RV myocardial blood flow. ${ }^{19}$ Indeed, as anticipated, at a baseline heart rate in this animal, blood flow to myocardium supplied by the anomalous LCA was greater than blood flow to myocardium supplied by the RCA (Figure 1, B). This finding and a normal electrocardiogram at rest suggested that myocardial blood flow was not impaired at baseline in this animal.

In normal mammals during exercise, LV blood flow exceeds RV blood flow by up to $20 \% .{ }^{19}$ Yet, as heart rate increased in this animal, blood flow to LCA myocardium was $20 \%$ lower than in RCA myocardium (Figure 1, B), and electrocardiographic changes were noted that were consistent with LV myocardial ischemia (Figure 1, $A$ ). These findings suggested that the anomalous LCA was unable to provide sufficient coronary flow to meet elevated LV myocardial metabolic demand.

Normally, the majority of coronary blood flow occurs during diastole. Systolic squeezing of the myocardium collapses coronary arterioles, and it is not until diastole when the heart relaxes that coronary resistance decreases and blood flow increases. In normal animals, the transition to arterial hypertension increases coronary blood flow during all phases of the cardiac cycle. However, in the present animal, arterial hypertension increased coronary resistance and decreased LCA blood flow during systole and beginning diastole. This pattern was consistent with aortic root compression-it was not until mid-diastole, after aortic recoil, that coronary resistance decreased (Figure 1,E), and mean diastolic coronary flow increased appropriately (Figure 1,D). These findings suggested that compression of the anomalous LCA between the aortic root and the pulmonary trunk produced myocardial ischemia.

It was not possible to evaluate the relative contributions from compression between the great vessels, the slit ostium, or unfavorable geometry of the LCA to the measured changes in coronary resistance. Of note, the pattern of systolic and early diastolic increase in resistance and decrease in flow were consistent with the timing of aortic root and pulmonary trunk expansion. Therefore, it cannot be ruled out that circumferential expansion of the aorta caused a dynamic narrowing of the slit opening, which may have contributed to increased coronary resistance and impairment of coronary blood flow.

\section{Inflammatory-Mediated Ischemic Myocardial Remodeling}

Histologic changes consistent with ischemic, inflammatorymediated myocardial remodeling were noted globally throughout the heart. In the left ventricle, repeated, brief coronary flow impairment during normal elevations of myocardial metabolic demands may have produced mild ischemic 
myocardial injury. The classic pattern of patchy fibrosis and hypertrophy of remaining myocytes was observed. Likewise, increased capillary density and collateralization in LV myocardium were present. Capillary density is normally uniform throughout the heart. ${ }^{20}$ Therefore, this finding further supported ischemia-induced injury and neovascularization. ${ }^{21}$

In response to ischemic myocardial injury, splenic reservoir mononuclear cells exit the spleen en masse and accumulate throughout the heart to participate in postinfarction myocardial remodeling. ${ }^{22}$ These potent undifferentiated inflammatory cells play an important role in orchestrating the construction and maintenance of the myocardial scar, but also affect myocardial remodeling in the ischemic penumbra and in distant myocardium. In the present animal, TUNEL staining demonstrated abnormal numbers of apoptotic nuclei throughout the heart. Myocyte apoptosis is rare in the myocardium. Consequently, the quantity and proximity to vasculature (Figure $2, H$ ) suggested that many of the apoptotic nuclei were transient immunologic cells that had extravasated to participate in ischemic, inflammatory-mediated myocardial remodeling. ${ }^{23}$

\section{Clinical Implications}

Our findings support the consistent association between anomalous coronary arteries and sudden cardiac death. ${ }^{1-11}$ In these individuals, inadequate coronary flow during aerobic exercise may upset the myocardial oxygen supplydemand relationship. Likewise, chronic hypertension or acute hypertension during anaerobic exercise may decrease blood flow when the coronary courses between the great vessels. As a result, acute ischemia may trigger fatal arrhythmia, or episodic ischemia may produce a remodeled and electrically unstable myocardial substrate predisposed to life-threatening ectopy.

This study is especially salient for young athletes in whom coronary anomalies account for $17 \%$ of sudden cardiac deaths. ${ }^{10}$ The first manifestation in these asymptomatic individuals is typically sudden death. ${ }^{4,5}$ In symptomatic patients in whom this anomaly is identified, it is widely accepted that corrective surgery should not be delayed. ${ }^{3,7}$ However, there is not a consensus that all patients with this anomaly should undergo operative therapy. ${ }^{8,9,11}$ Our data demonstrate that an anomalous coronary artery that courses between the great vessels may result in inadequate regional myocardial blood flow. Therefore, these findings support surgical repair in patients with acceptable operative risk.

These are the first empiric data to document myocardial ischemia with an anomalous LCA from the right sinus of Valsalva during increased myocardial metabolic demands. Ischemic myocardial remodeling was present. These findings support early surgical repair of this anomaly. If elective surgery is declined, pharmacologic therapy, such as betaadrenergic blockade, may be considered to decrease myocardial metabolic demands. Appropriate management of hypertension may also decrease the likelihood of myocardial ischemia.

\section{Limitations}

A limitation was that this study was performed in a single calf. Coronary anomalies are rare, and an anomalous LCA from the right sinus of Valsalva has never been described or studied in an experimental large animal. As such, it was not feasible to increase the sample size.

\section{CONCLUSIONS}

Notwithstanding this limitation, the current study contains several strengths that included a clinically relevant large-animal model, multiple measurements of coronary blood flow, regional myocardial blood flow, systemic hemodynamics, and electrocardiography during different physiologic conditions and multiple histologic measurements of myocardial remodeling. Each type of data demonstrated pathology consistent with an ischemic cause and support previously proposed hypotheses about the mechanism of sudden death with an anomalous coronary artery from the right sinus of Valsalva.

The authors thank Mark S. Slaughter, MD, Robert D. Acland, MD, Michael A. Sobieski, RN, CCP, Kenneth R. Brittian, and the University of Louisville veterinary staff for assistance.

\section{References}

1. Yamanaka O, Hobbs RE. Coronary artery anomalies in 126,595 patients undergoing coronary arteriography. Cathet Cardiovasc Diagn. 1990;21:28-40.

2. Angelini P. Coronary artery anomalies: an entity in search of an identity. Circulation. 2007;115:1296-305

3. Kayalar N, Burkhart HM, Dearani JA, Cetta F, Schaff HV. Congenital coronary anomalies and surgical treatment. Congenit Heart Dis. 2009;4: 239-51.

4. Basso C, Maron BJ, Corrado D, Thiene G. Clinical profile of congenital coronary artery anomalies with origin from the wrong aortic sinus leading to sudden death in young competitive athletes. J Am Coll Cardiol. 2000;35 1493-501.

5. Eckart RE, Scoville SL, Campbell CL, Shry EA, Stajduhar KC, Potter RN, et al Sudden death in young adults: a 25 -year review of autopsies in military recruits. Ann Intern Med. 2004;141:829-34.

6. Taylor AJ, Byers JP, Cheitlin MD, Virmani R. Anomalous right or left coronary artery from the contralateral coronary sinus: "high-risk" abnormalities in the initial coronary artery course and heterogeneous clinical outcomes. Am Heart J. 1997; 133:428-35.

7. Gulati R, Reddy VM, Culbertson C, Helton G, Suleman S, Reinhartz O, et al. Surgical management of coronary artery arising from the wrong coronary sinus, using standard and novel approaches. J Thorac Cardiovasc Surg. 2007;134: 1171-8.

8. Frommelt PC, Frommelt MA, Tweddell JS, Jaquiss RD. Prospective echocardiographic diagnosis and surgical repair of anomalous origin of a coronary artery from the opposite sinus with an interarterial course. J Am Coll Cardiol. 2003; 42:148-54.

9. Gersony WM. Management of anomalous coronary artery from the contralateral coronary sinus. J Am Coll Cardiol. 2007;50:2083-4.

10. Maron BJ, Thompson PD, Ackerman MJ, Balady G, Berger S, Cohen D, et al Recommendations and considerations related to preparticipation screening for cardiovascular abnormalities in competitive athletes: 2007 update: a scientific statement from the American Heart Association Council on Nutrition, Physical Activity, and Metabolism: endorsed by the American College of Cardiology Foundation. Circulation. 2007;115:1643-55. 
11. Krasuski RA, Magyar D, Hart S, Kalahasti V, Lorber R, Hobbs R, et al. Long-term outcome and impact of surgery on adults with coronary arteries originating from the opposite coronary cusp. Circulation. 2011;123: 154-62.

12. Bartoli CR, Okabe K, Akiyama I, Coull B, Godleski JJ. Repeat microsphere delivery for serial measurement of regional blood perfusion in the chronically instrumented, conscious canine. J Surg Res. 2008;145:135-41.

13. Bartoli CR, Wellenius GA, Coull BA, Akiyama I, Diaz EA, Lawrence J, et al. Concentrated ambient particles alter myocardial blood flow during acute ischemia in conscious canines. Environ Health Perspect. 2009;117:333-7.

14. Seeberger MD, Skarvan K, Buser P, Brett W, Rohlfs R, Erne JJ, et al. Dobutamine stress echocardiography to detect inducible demand ischemia in anesthetized patients with coronary artery disease. Anesthesiology. 1998;88: 1233-9.

15. Bartoli CR, Wilson GC, Giridharan GA, Slaughter MS, Sherwood LC, Spence PA, et al. A novel subcutaneous counterpulsation device: acute hemodynamic efficacy during pharmacologically induced hypertension, hypotension, and heart failure. Artif Organs. 2010;34:537-45.

16. Koenig SC, Woolard C, Drew G, Unger L, Gillars K, Ewert D, et al. Integrated data acquisition system for medical device testing and physiology research in compliance with good laboratory practices. Biomed Instrum Technol. 2004;38: 229-40.
17. Schroeder MJ, Perreault B, Ewert DL, Koenig SC. HEART: an automated beat-to-beat cardiovascular analysis package using Matlab. Comput Biol Med. 2004;34:371-88.

18. Bartoli CR, Brittian KR, Giridharan GA, Koenig SC, Hamid T, Prabhu SD. Bovine model of doxorubicin-induced cardiomyopathy. J Biomed Biotechnol. 2011; 2011:758736.

19. Duncker DJ, Bache RJ. Regulation of coronary blood flow during exercise. Physiol Rev. 2008;88:1009-86.

20. Anversa P, Li P, Sonnenblick EH, Olivetti G. Effects of aging on quantitative structural properties of coronary vasculature and microvasculature in rats. Am J Physiol. 1994;267(3 Pt 2):H1062-73.

21. Kajstura J, Zhang X, Reiss K, Szoke E, Li P, Lagrasta C, et al. Myocyte cellular hyperplasia and myocyte cellular hypertrophy contribute to chronic ventricular remodeling in coronary artery narrowing-induced cardiomyopathy in rats. Circ Res. 1994;74:383-400.

22. Swirski FK, Nahrendorf M, Etzrodt M, Wildgruber M, Cortez-Retamozo V, Panizzi P, et al. Identification of splenic reservoir monocytes and their deployment to inflammatory sites. Science. 2009;325:612-6.

23. Hamid T, Gu Y, Ortines RV, Bhattacharya C, Wang G, Xuan YT, et al. Divergent tumor necrosis factor receptor-related remodeling responses in heart failure: role of nuclear factor-kappaB and inflammatory activation. Circulation. 2009;119: 1386-97. 